\title{
The Paediatric Observation Priority Score: A System to Aid Detection of Serious Illness and Assist in Safe Discharge
}

\author{
Damian Roland ${ }^{1,2}$, Gareth Lewis ${ }^{1}$, Peter Fielding ${ }^{1}$, Carla Hakim ${ }^{1}$, Alexandra Watts ${ }^{1}$, \\ Ffion Davies ${ }^{1}$ \\ ${ }^{1}$ Paediatric Emergency Medicine Leicester Academic (PEMLA) Group, Children's Emergency Department, \\ Leicester Royal Infirmary, Leicester, UK \\ ${ }^{2}$ SAPPHIRE Group, Health Sciences, Leicester University, Leicester, UK \\ Email: dr98@le.ac.uk
}

Received 16 March 2016; accepted 11 June 2016; published 14 June 2016

Copyright (C) 2016 by authors and Scientific Research Publishing Inc.

This work is licensed under the Creative Commons Attribution International License (CC BY).

http://creativecommons.org/licenses/by/4.0/

(c) (i) Open Access

\begin{abstract}
The Paediatric Observation Priority Score (POPS) is a bespoke assessment tool for use in Paediatric Emergency Departments incorporating traditional physiological parameters alongside more subjective observational criteria. Initial performance characteristics of POPS were analysed in a convenience sample of $\mathbf{9 3 6}$ presentations to ED. Triage on the basis of gut instinct parameters identified an additional 261 patients deemed of lowest acuity compared to analysis by physiology scores. Resource consumption increased with increasing acuity on presentation. POPS shows promise in assisting in the assessment process of children presenting to Emergency Departments. Inclusion of subjective triage criteria helps contextualise the physiological parameter scoring by using the experience of staff conducting triage. Initial interpretation of presenting physiology gives a more informed assessment of initial acuity, and thus is better able to identify a child who can be safely managed in the community. The system also allows for rapid detection of those most unwell.
\end{abstract}

\section{Keywords}

Emergency Department, Children and Young People, Paediatric, Triage, Patient Discharge, Paediatric Early Warning

\section{Introduction}

Children with serious illness can be difficult to spot, especially for non-experienced staff. Healthcare systems

How to cite this paper: Roland, D., Lewis, G., Fielding, P., Hakim, C., Watts, A. and Davies, F. (2016) The Paediatric Observation Priority Score: A System to Aid Detection of Serious Illness and Assist in Safe Discharge. Open Journal of Emergency Medicine, 4, 38-44. http://dx.doi.org/10.4236/ojem.2016.42006 
face a challenge to select these children from an ever increasing pool of attendances to emergency and urgent care providers [1]. Determining the relative acuity of children presenting to emergency and urgent care environments has traditionally been on the basis of triage. This is a time based system and does not always accurately reflect the illness or eventual disposition of the patient [2]. In adult practice Early Warnings Scores (EWS) have been used to detect serious illness but there are no validated systems for children. A recent retrospective study employing ward based systems in Children's Emergency Departments (ED) demonstrated their ability to detect children requiring intensive care but not those requiring admission [3]. Given the majority of children presenting to emergency or urgent care services are likely to be discharged, systems employed need to be able to assist in the determination of both the most sick and those who are well enough to go home.

Locally we created, using current evidence and the experience of senior paediatric emergency clinicians; the Paediatric Observation Priority Score (POPS). We noted the only previous work in this field by Bradman and Maconochie [4] and aimed to expand on their initial findings that an early warning score system was of limited value in predicting admission but was useful in determining discharge. POPS is a physiological and observational scoring system (range 0 - 16) designed for use by health care professionals of varying clinical experience at initial assessment in an urgent or emergency care setting.

It consists of 8 domains (oxygen saturations, level of alertness, extent of breathing difficulty, background history, nurse gut feeling, heart rate, respiratory rate and temperature) each graded 0,1 or 2 to give a total score of 16 (Appendix). Physiological sub-scores carry a total of 10 points, with gut instinct and appearance sub-scores (level of alertness, extent of breathing difficulty and gut feeling) contributing a further 6 potential points. The parameters were chosen based on APLS guidance and their utilisation in other scoring systems. The visual style was based on feedback from nurses over a 1 month period, constantly refined based on feedback.

A small pilot phase in 100 patients (presented at a regional paediatric meeting) demonstrated acceptability and feasibility. This, the first stage of an ongoing validation process, describes the initial prospective evaluation.

\section{Methods}

Leicester Royal Infirmary is a paediatric tertiary centre with a dedicated Children's ED. Since POPS was introduced in the ED, data has been collected prospectively on the demographics of attendees, their initial POPS on presentation, resource consumption in ED and eventual outcome from the department. All patients attending the paediatric ED receive initial triage assessment by a trained nurse competent in the use of POPS.

We identified the above data parameters in a randomly selected sample of patients who attended the department between 08/01/09-07/01/11. A database of 936 patients aged 0 - 15 years was constructed. The initial POPS on triage was used to determine acuity on presentation to ED. Alongside total POPS, the constituent physiology and gut-instinct sub-scores were recorded for each presentation.

This database was interrogated to determine whether the inclusion of triage gut instinct parameters in POPS augment its ability to identify those patients of low presenting acuity who may be suitable for discharge home or to community care teams. The length of stay in department and subsequent ED resource consumption (imaging modality, IV access, bloods, analgesia, oxygen, nebuliser, fluid and antibiotic utilisation) was also investigated. Patient outcome in ED (i.e. discharge destination), duration of stay in department and resource consumption was therefore analysed as a function of total and constituent POPS sub-scores. The data was arranged in order of descending patient acuity as analysed by total and sub-score constituent POPS. Physiology sub-scores were grouped into narrow ranges of values. A pragmatic assumption was made that a physiology sub-score of 1 - 2 out of a possible 10 is equivalent to a gut instinct sub-score of 1 out of 6, and that 3 - 4 out of 10 (physiology) was equivalent to 2 out of 6 (gut instinct) and so on; such that 6 equivalent degrees of presenting acuity were assigned for physiology and gut-instinct sub-scores to facilitate comparison. A score of zero in either sub-score was deemed equivalent.

Line charts and histograms collating data for the number of presentations for each relative degree of acuity were created in the above contexts. Microsoft Excel software (2010) was used to produce all graphs.

\section{Results}

The distribution of initial triage POPS of the attendees is shown in Figure 1. 32\% of all patients had a POPS of $0,37 \%$ had a POPS of $1-2$ and $21 \%$ of $3-4$.

Average duration of stay in ED for all patients was 137.2 minutes regardless of POPS and eventual outcome. 


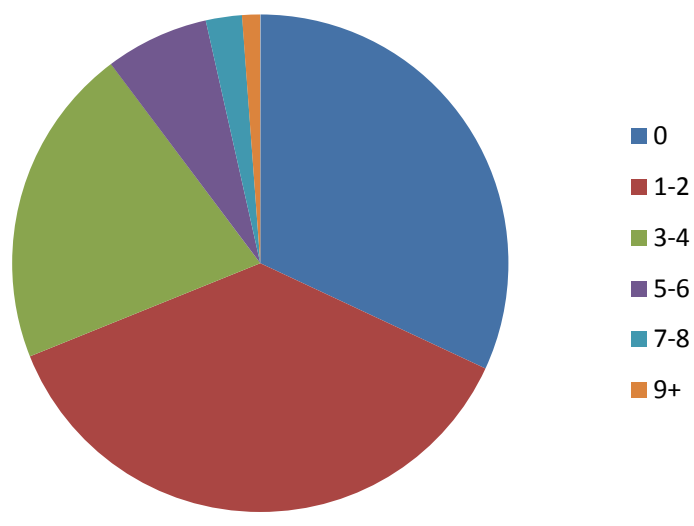

Figure 1. Distribution of POPS summated for all age groups of Emergency Department attendees.

Taking this into account, Figure 2 shows variation by POPS from the overall average time spent in ED.

Eventual outcome of each patient was analysed by initial POPS at time of presentation (Table 1). Included in the table below is one child who presented with a triage POPS of 12 who went to PICU (paediatric intensive care unit). This child is categorised under Children's Admissions Unit (CAU)/Ward, as it represents an admission to a hospital bed.

$77.8 \%(185+47 / 298)$ of children with a triage POPS of 0 were discharged from ED. Ignoring triage POPS and taking the population as a whole; $55 \%$ of all patients were discharged home direct from ED; a figure which rises to $64 \%$ discharged from ED when those amenable to re-direction are considered. Table 2 shows outcomes as analysed by physiology and instinctive sub-scores.

Figure 3 shows that both sub-scores perform similarly in terms of predicting likely discharge disposition for a patient attending with each respective POPS score. This figure assumes that a score of zero for POPS, physiology and gut instinct are equivalent (point $\mathrm{A}$ ), as are a score of 1 - 2 for physiology and a score of 1 for gut instinct (point B) and so on.

Figure 4 shows the average number of interventions per patient in the ED; organised by way of their POPS score and eventual outcome in terms of clinical management.

\section{Discussion}

POPS provides a methodology of classifying acuity in a Children's Emergency Department which is pragmatic, supports disposition and equates to resource utilisation. In this initial validation cohort the majority of presentations were of children of low clinical acuity when analysed by POPS. 69\% of all attendees had a total POPS of 2 or less.

By incorporating gut instinct and appearance factors into the triage scoring of patients, we are able to expand the evidence we have to contextualise the clinical management decisions that we make. Triaging patients on the basis of their gut instinct and appearance sub-score identified an additional 261 patients of the 936 sampled who were of the lowest stratification of acuity and therefore potentially suitable for discharge from emergency care. Further work is needed to determine whether these observational characteristics can be used in isolation.

When considering duration of stay in ED (Figure 2), it is apparent that those with a total POPS score of 2 - 7 appear to stay in ED for longer than the average waiting time. This may be because they are more unwell than those with lower POPS and require stabilisation, or require more investigations or assessment prior to decision making with regards to discharge disposition and outcome. It is noted those with higher total POPS scores of 8 10 also stay in the department for less time than average. A potential explanation would be that these patients are referred efficiently if it is apparent that they require hospital admission, although the low patient numbers exhibiting this level of acuity demonstrate a need for further delineate this pattern.

It is a reasonable assumption that those patients of higher clinical acuity will require the most resources in terms of investigation and intervention. Figure 4 shows judicious use of ED investigation in those patients who are immediately re-directed to urgent care centres and other community care teams. As anticipated, those patients who are discharged from ED consume fewer resources than those who are admitted, whilst the average 


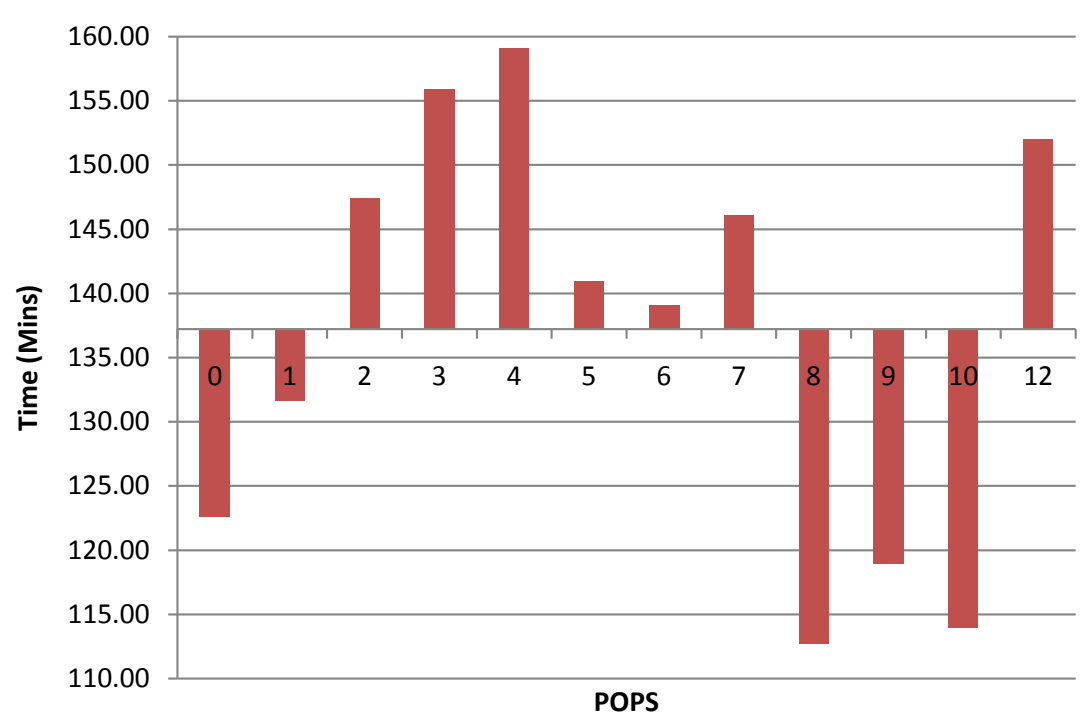

Figure 2. Average time in department by POPS relative to overall average waiting time.

Table 1. Outcome of 936 children presenting to the Emergency Department by their initial POPS. The urgent care centre is run by General Practitioners (Family Doctors) and indicates the child has been discharged into primary care.

\begin{tabular}{|c|c|c|c|c|c|}
\hline POPS & Total Patients & Home & Assessment Unit/Ward & Urgent Care Centre & Return Post Discharge \\
\hline 0 & 298 & 185 & 66 & 47 & 16 \\
\hline $1-2$ & 346 & 210 & 106 & 30 & 22 \\
\hline $3-4$ & 196 & 98 & 91 & 7 & 15 \\
\hline $5-6$ & 63 & 17 & 44 & 2 & 5 \\
\hline $7-8$ & 22 & 2 & 18 & 1 & 4 \\
\hline $9+$ & 11 & 1 & 9 & 0 & 1 \\
\hline Total & 936 & 513 & 334 & 87 & 63 \\
\hline
\end{tabular}

Table 2. Outcome of all 936 children presenting to ED by their physiology and gut instinct sub-scores of POPS. Physiology (P) sub-scores in white, out of 10 . Gut Instinct (I) sub-scores in grey, out of 6. A = P0 G0, B = P1-2 G1, C = P3-4 G2, D = P5-6 G3, E = P7-8 G4, F = P9-10 G5-6.

\begin{tabular}{|c|c|c|c|c|c|}
\hline POPS Sub-Score & Total Patients & Home & CAU/Ward & UCC/CRAFT & Return Post Discharge \\
\hline \multirow{2}{*}{ A } & 358 & 219 & 89 & 50 & 19 \\
\hline & 619 & 375 & 167 & 77 & 39 \\
\hline \multirow{2}{*}{ B } & 394 & 221 & 142 & 31 & 27 \\
\hline & 182 & 96 & 81 & 5 & 12 \\
\hline \multirow{2}{*}{$\mathrm{C}$} & 160 & 69 & 85 & 5 & 14 \\
\hline & 86 & 36 & 46 & 4 & 6 \\
\hline \multirow{2}{*}{$\mathrm{D}$} & 23 & 4 & 18 & 1 & 3 \\
\hline & 26 & 4 & 22 & 0 & 3 \\
\hline \multirow{2}{*}{ E } & 1 & 0 & 0 & 0 & 0 \\
\hline & 16 & 1 & 14 & 1 & 2 \\
\hline \multirow{2}{*}{$\mathrm{F}$} & 0 & 0 & 0 & 0 & 0 \\
\hline & 7 & 1 & 4 & 0 & 1 \\
\hline Totals & 936 & 513 & 334 & 87 & 63 \\
\hline
\end{tabular}




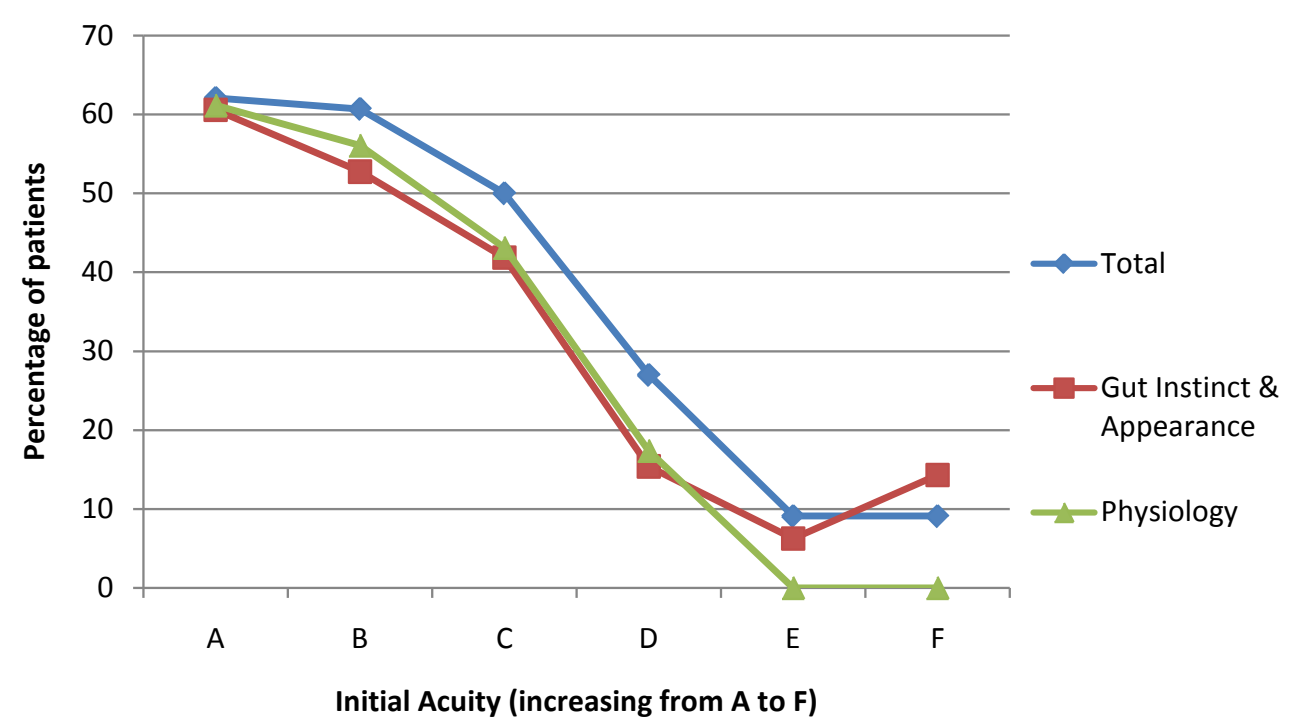

Figure 3. Percentage of patients discharged home from ED by their total and constituent POPS.

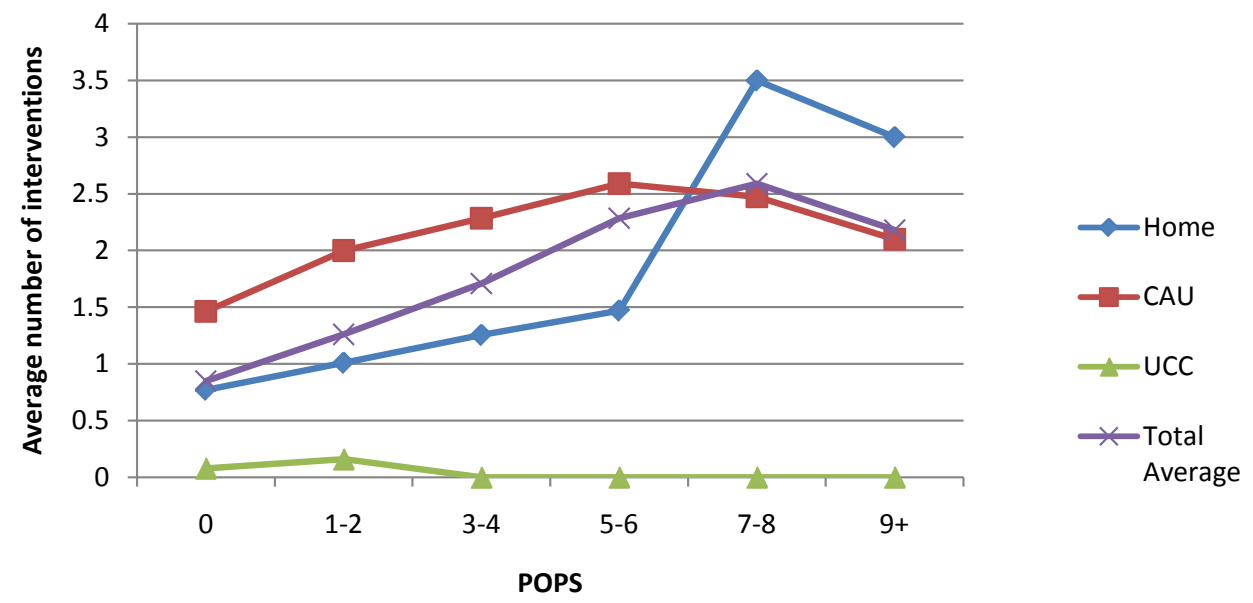

Figure 4. Average number of interventions per person attending ED by POPS and outcome.

number of investigations or interventions per person increases with increasing clinical acuity on presentation.

This study is of relatively old data due to the lead author undertaking a nationally funded fellowship in another research area during the years 2010-13. Results are consistent with an ongoing study [5] and another group who have utilised POPS in their own department [6]. POPS remains one of the few published Emergency Department specific scoring systems [7].

\section{Conclusions}

The premise of the Paediatric Observation Priority Score is simple (POPS): it represents a bespoke method of identifying children with potentially serious illnesses while at the same time safely supporting staff in redirecting or discharging those who do not need ongoing care. This allows for the most sick and most well children to be clearly identified early in the patient journey. POPS has demonstrated a functional ability to aid health care professionals' decision making. The results of this work have led to a larger scale study (United Kingdom Clinical Research Network study number 11532) and its deployment in other centres around the United Kingdom.

With further investigation and refinement we believe POPS will reduce risk-averse strategies of referring all children of 'potential concern' for specialist paediatric assessment. This overloads an already stretched out of hours system, and leads to unnecessary hospital admissions - which is poor practice from both a resource and a patient/family care point of view, while at the same time ensuring the most unwell patients are recognised and 
acted on in a prompt fashion.

\section{References}

[1] Gill, P., Goldacre, M., Mant, D., et al. (2013) Increase in Emergency Admissions to Hospital for Children Aged under 15 in England, 1999-2010: National Database Analysis. Archives of Disease in Childhood, 98, 32-34. http://dx.doi.org/10.1136/archdischild-2012-302383

[2] Van Veen, M., Steyerberg, E.W., Ruige, M., van Meurs, A.H., Roukema, J., van der Lei, J., et al. (2008) Manchester Triage System in Paediatric Emergency Care: Prospective Observational Study. BMJ, 337, a1501. http://dx.doi.org/10.1136/bmj.a1501

[3] Seiger, N., Maconochie, I. and Oostenbrink, R. (2013) Validity of Different Pediatric Early Warning Scores in the Emergency Department. Pediatrics, 132, e841-e850.

[4] Bradman, K. and Maconochie, I. (2008) Can Paediatric Early Warning Score Be Used as a Triage Tool in Paediatric Accident and Emergency? European Journal of Emergency Medicine, 15, 359-360. http://dx.doi.org/10.1097/MEJ.0b013e3283026208

[5] Roland, D., Davies, F. and Coats, T. (2014) The Paediatric Observation Priority Score (POPS): Outcomes of 24000 Patients. Archives of Disease in Childhood, 99, A24. http://dx.doi.org/10.1136/archdischild-2014-307384.77

[6] Rowland, A., Cotterill, S., Lees, H., et al. (2014) The Paediatric Observation Priority Score (POPS): A More Accurate Predictor of Admission Risk from the Emergency Department than the Manchester Children's Early Warning System (ManChEWS). Archives of Disease in Childhood, 99, A8. http://dx.doi.org/10.1136/archdischild-2014-306237.16

[7] Challen, K. and Roland, D. (2016) An Early Warning Scores: A Health Warning. EMJ Published Online First 4 January 2016. 


\section{Appendix}

Paediatric Observation Priority Score (POPS) Chart

This chart is not a substitute for good clinical judgement and any concerns about the condition of a child should be brought to the attention of a senior nurse or doctor

\begin{tabular}{|c|c|c|c|c|c|c|}
\hline Age & Score & 2 & 1 & 0 & 1 & 2 \\
\hline Any & Sats & $<90 \%$ & $90-94 \%$ & $>95 \%$ & $90-94 \%$ & $<90 \%$ \\
\hline Any & Breathing & Stridor & $\begin{array}{l}\text { Audible grunt or } \\
\text { wheeze }\end{array}$ & No distress & $\begin{array}{l}\text { Mild or Moderate } \\
\text { Recession }\end{array}$ & $\begin{array}{c}\text { Severe } \\
\text { Recession }\end{array}$ \\
\hline Any & AVPU & Pain & Voice & Alert & Voice & Pain \\
\hline Any & $\begin{array}{l}\text { Gut } \\
\text { Feeling }\end{array}$ & $\begin{array}{l}\text { High level } \\
\text { concern }\end{array}$ & $\begin{array}{l}\text { Low level } \\
\text { concern }\end{array}$ & Well & $\begin{array}{l}\text { Low level } \\
\text { concern }\end{array}$ & $\begin{array}{l}\text { Child looks } \\
\text { unwell }\end{array}$ \\
\hline Any & Other & $\begin{array}{l}\text { Oncology } \\
\text { Patient }\end{array}$ & $\begin{array}{l}\text { Significant } \\
\mathrm{PMH}^{*}\end{array}$ & & $\begin{array}{l}\text { Significant } \\
\mathrm{PMH}^{*}\end{array}$ & $\begin{array}{l}\text { Congenital heart } \\
\text { disease }\end{array}$ \\
\hline \multirow{3}{*}{$0-1$} & Pulse & $<90$ & $90-109$ & $110-160$ & $161-180$ & $180+$ \\
\hline & RR & $<25$ & $25-29$ & $30-40$ & $41-50$ & $50+$ \\
\hline & Temp & $<35^{\circ}$ & $35-35.9^{\circ}$ & $36-37.5^{\circ}$ & $37.6-39^{\circ}$ & $39^{\circ}+$ \\
\hline \multirow{3}{*}{$1-2$} & Pulse & $<90$ & $90-99$ & $100-150$ & $151-170$ & $170+$ \\
\hline & RR & $<20$ & $20-24$ & $25-35$ & $36-50$ & $50+$ \\
\hline & Temp & $<35^{\circ}$ & $35-35.9^{\circ}$ & $36-38.4^{\circ}$ & $38.5-40^{\circ}$ & $40^{\circ}+$ \\
\hline \multirow{3}{*}{$2-5$} & Pulse & $<80$ & $80-94$ & $95-140$ & $141-160$ & $160+$ \\
\hline & RR & $<20$ & $20-24$ & $25-30$ & $31-40$ & $40+$ \\
\hline & Temp & $<35^{\circ}$ & $35-35.9^{\circ}$ & $36-38.4^{\circ}$ & $38.5-40^{\circ}$ & $40^{\circ}+$ \\
\hline \multirow{3}{*}{$5-12$} & Pulse & $<70$ & $70-79$ & $80-120$ & $121-150$ & $150+$ \\
\hline & RR & $<15$ & $15-19$ & $20-25$ & $26-40$ & $40+$ \\
\hline & Temp & $<35^{\circ}$ & $35-35.9^{\circ}$ & $36-38.4^{\circ}$ & $38.5-40^{\circ}$ & $40^{\circ}+$ \\
\hline \multirow{3}{*}{$13-16$} & Pulse & $<50$ & $50-60$ & $60-100$ & $100-110$ & $110+$ \\
\hline & RR & $<12$ & $12-14$ & $15-20$ & $20-25$ & $25+$ \\
\hline & Temp & $<35^{\circ}$ & $35-35.9^{\circ}$ & $36-38.4^{\circ}$ & $38.5-40^{\circ}$ & $40^{\circ}+$ \\
\hline
\end{tabular}

\begin{tabular}{|c|c|}
\hline $\begin{array}{c}\text { Total } \\
\text { Score }\end{array}$ & Priority \\
\hline $0-1$ & \\
$2-3$ & \\
$4-7$ & \\
$8+$ & $\begin{array}{c}\text { Immediate } \\
\text { review }\end{array}$ \\
\hline
\end{tabular}

Any child scoring above 8 should be considered for transfer to resus

*Significant PMH
includes:
- Ex-premature
- Syndromic
conditions
- Cardiac problems
- Asthma
- Diabetes
- Long term steroids
- All other chronic
conditions

POPS is copyright (creative commons attribution non-commercial sharealike 4.0) Dr Damian Roland and Dr Ffion Davies 2010 This is version 1.2 August 2015 